\title{
The Role of Whole-Process Quality Management Mode in the Self-Protection and Safety of First-Line Medical Staff of COVID-19
}

\author{
Juan Ding1*, Yujiao Yan' ${ }^{2 *}$ Mo Fu ${ }^{1 \#}$ \\ ${ }^{1}$ Nursing Department, Jingzhou Central Hospital, Jingzhou, China \\ ${ }^{2}$ Department of Medicine, Yangtze University, Jingzhou, China \\ Email:"308722048@qq.com
}

How to cite this paper: Ding, J., Yan, Y.J. and Fu, M. (2021) The Role of Whole-Process Quality Management Mode in the Self-Protection and Safety of First-Line Medical Staff of COVID-19. Yangtze Medicine, 5, 61-69. https://doi.org/10.4236/ym.2021.51007

Received: April 7, 2020

Accepted: January 11, 2021

Published: January 14, 2021

Copyright $\odot 2021$ by author(s) and Scientific Research Publishing Inc. This work is licensed under the Creative Commons Attribution International License (CC BY 4.0).

http://creativecommons.org/licenses/by/4.0/

\begin{abstract}
At present, the COVID-19 has spread all over the world. In the face of sudden outbreak, it has brought great challenges to the management of nosocomial infection prevention and control. In this battle, how to do well the prevention and control of hospital epidemic situation scientifically and reasonably is the key to hospital management and an important link to ensure the safety of first-line medical staff. Through the clinical practice of our hospital to combat the COVID-19 epidemic situation, we summarize the key points and functions of quality management, and provide management strategies for domestic and foreign countries.
\end{abstract}

Keywords

COVID-19, Quality Management, Safety

\section{Introduction}

Since the outbreak of the new type of coronavirus pneumonia (COVID-19), there have been multiple confirmed cases of first-line medical staff across the country. The incidence of medical staff infections in medical institutions is higher than usual, which has aroused public concern. Medical staff infection not only causes damage to the physical and mental health of medical staff, but also has a huge impact on the treatment and prevention of COVID-19 [1]. How to ensure the safety of medical personnel's own protection and ensure "zero infection" is very important. By adopting a full-process quality management model, our hospital has ensured the self-protection safety of front-line medical staff in ${ }^{\star} J$ Juan Ding and Yujiao Yan are considered joint first authors. 
the COVID-19 epidemic and achieved the goal of "zero infection". Relevant research shows that [2] [3], the whole-process quality management model can effectively reduce nursing errors and medical disputes, standardize the work behavior of nursing staff, and improve the level of scientific and standardized management in hospitals. Chen Chunxi et al. [4]. found in the whole process of quality management of the delivery of sterile materials in the central supply room that it was found that the adoption of full quality management measures can reduce the re-contamination of sterile materials and control hospital infections. In 2003, Shanghai Infectious Diseases Hospital, as a designated hospital for the treatment of suspected and confirmed domestic SARS cases found in Shanghai, achieved good results in successfully rescuing SARS patients and medical staff with zero infection by strengthening the practice of medical quality management ${ }^{[5]}$. In the face of sudden outbreaks, the establishment of scientific management models and management methods is imminent.

Our hospital is a prefecture-level regional medical center in Hubei Province. On January 21, 2020, it was designated as the hot spot medical institution and designated treatment medical institution of Jingzhou COVID-19, and designated treatment hospital for critically ill patients, through the whole process quality management mode was established including management team, system specification, emergency plan drills, professional training, process improvement and quality supervision and other measures more than (as shown in Figure 1), to achieve the goal "zero infection". The report is as follows.

\section{Practice}

\subsection{Set up COVID-19 Protective Safety Management Team}

Thecovid-19 protection safety management team is composed of members of the head of hospital infection management department, the chief of medical department, the director of the quality control office, the director of the nursing

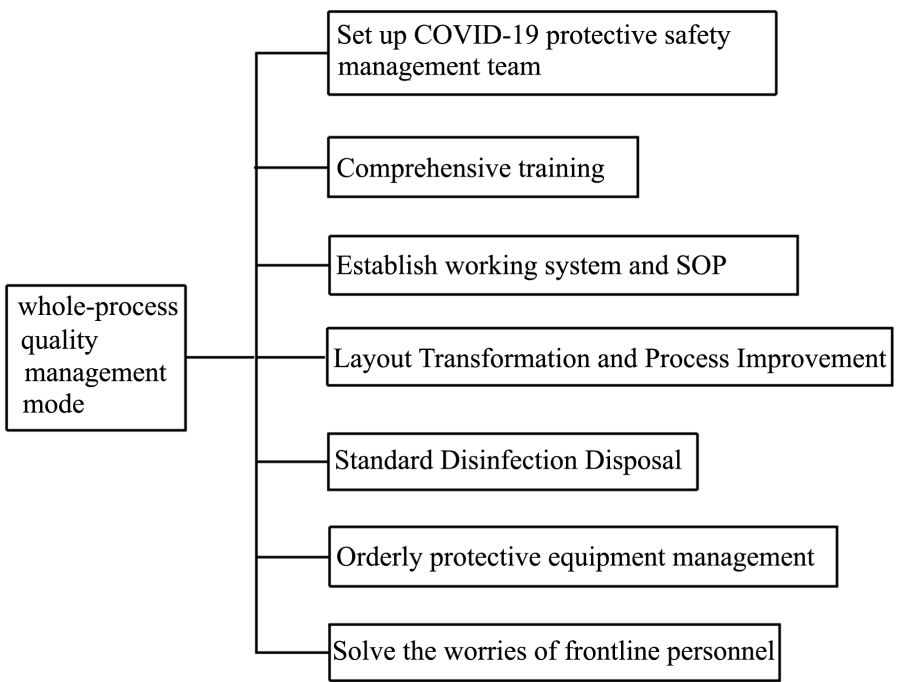

Figure 1. COVID-19 whole-process quality management model. 
department, the chief of public health department, the chief of material supply office and the chief of equipment department, overall responsibility for work guidance, supervision, training, management, etc. Unified management, strengthen cooperation, sincere cooperation, and clear responsibilities: the Department of Hospital Infection Management is responsible for the work guidance and quality supervision of COVID-19 protection and safety; The medical department, quality control office, nursing department, etc. are responsible for the training, assessment and implementation of various rules and regulations of the new coronary pneumonia related knowledge; the public health department mainly conducts regular sampling and testing of the ward environment; the instrument department provides disinfection instruments, equipment maintenance and supply. The team members divided the work, contacted the person in charge of each link from point to point, understood the work needs, collected the problems, communicated with the WeChat group in time, and required a reply within 24 hours. The COVID-19 protective safety management team meets every 3 days to form a mechanism: 1) overall protection in critical isolation areas; 2) feedback the current problems in its operation; 3) propose proposed solutions and remedial measures for solvable problems; For problems that cannot be solved temporarily, all team members shall discuss and consult with each other. If other departments need to coordinate and solve or report to the hospital, the team leader shall coordinate and report to the hospital.

\subsection{Comprehensive Training}

\subsubsection{At the Beginning of the Epidemic, Basic Knowledge about COVID-19 Was Popularized}

On December 30th, the Health and Medical Committee of Wuhan City of our province issued the emergency notice of "Treatment of Unknown Virus Infected Pneumonia" for the first time, our hospital collected relevant information in a timely manner, and asked the hospital's medical staff to be more vigilant and better protected. Focus on training in hand hygiene, wearing masks, putting on and taking off protective clothing and isolation clothes, using goggles, procedures for entering and leaving the quarantine area, and disinfection and quarantine. Relevant standard operating procedures (SOP) have also been developed to facilitate the correct implementation of medical staff. At the same time, we conducted three-level training at the college level-area-department, including propaganda of prevention knowledge and special lectures. At the same time, the hospital experts were organized to answer questions and make doubts, so that the medical staff had a certain understanding of covid-19 in the early stage of the epidemic, and had a basic awareness of protection.

\subsubsection{During the Admission and Treatment Phase, Focus on Training in Prevention and Control Knowledge}

The main route of transmission of COVID-19 is through respiratory droplets and close contact, there is a possibility of aerosol transmission in a relatively closed environment when exposed to high concentrations of aerosol for a long 
time [6]. Therefore, strict disinfection and isolation is an important means to cut off the transmission path. Our hospital conducted comprehensive training on nosocomial infection prevention and control of COVID-19, placement and management of COVID-19 patients, disinfection treatment measures of COVID-19 and personal protection of medical staff through batch training, WeChat, Ding Dingyun classroom and other online learning. The training and interpretation of the "COVID-19 Diagnosis and Treatment Plan" (first to seventh edition) and "COVID-19 Prevention and Control Plan" (first to fifth edition) issued by the National Health and Health Commission in a timely manner, constantly updated knowledge and ideas. At the same time, training and examination were conducted on nasopharyngeal swab collection technology, closed sputum aspiration technology, invasive mechanical ventilation technology, non-invasive ventilator technology, high-flow oxygen inhalation technology, etc., to ensure everyone to pass the exam.

\subsection{Establish Working System and SOP}

\subsubsection{Formulate Specifications}

When the first suspected case of COVID-19 was admitted to our hospital on January 16, 2020, the safety control panel meeting immediately, successively formulated the "The Working System for Critical Isolation Wards", "The Transfer Process for Suspected Patients", "The Daily Disinfection Measures for Isolation Wards", "The Final Disinfection Measures for Isolation Wards", "System for Application and Standardized Use of Protective Equipment during Epidemic Period", "Disposal of fever patients in general wards (rooms)", "Logistics Support System" and other regulations, "Methods of putting on and taking off protective clothing", "Methods of protective eyepiece fogging" and other SOPs. On February 16, after the establishment of Jingzhou COVID-19 Severe Care Center, according to the new environment, the duties and procedures of each shift were rearranged to form the electronic file of "Jingzhou Central Hospital COVID-19 should know and should do", which is convenient to study at any time.

\subsubsection{Formulate Emergency Plan}

COVID-19 epidemic has a huge impact, patients withcovid-19 are not limited to fever clinics, any department is at risk of receiving new patients with covid-19 or exposing new type of coronavirus. According to the relevant normative guidelines, evaluate various emergencies in advance, and develop emergency response procedures based on local materials under the guidance of the hospital infection management department, including "The Emergency Response Plan for Damage to Protective Clothing", "Emergency Plan for Needle Injury", "Hypoglycemic Emergency Plan", "Prevention and Treatment Procedures of Head and Face Skin Injury Caused by Protective Appliances" and many other emergency plans. For example, in the "Emergency Treatment Plan for Protective Clothing Damage", once the protective clothing is damaged, medical personnel should immediately use $75 \%$ ethanol spray or quick-dry hand disinfectant to apply the damaged area 
(spraying or applying range is greater than 3 times the diameter of the damaged area), and inform Medical staff of the same shift, hand over work with the same staff, evacuate the quarantine area, take off protective equipment and work clothes according to the process, and shower and change clothes, re-wear protective equipment according to work needs and enter the quarantine area. The work should focus on the integrity of the protective clothing, timely detection of cracks or damage, to minimize the risk of infection. Timely and proper handling of the damage of protective clothing can effectively protect the life safety and health of frontline medical personnel.

\subsubsection{Supervise Implementation}

Effective implementation of rules and regulations is an important guarantee to prevent hospital infection and ensure orderly work. Each isolation area is equipped with a hospital infection administrator, who is responsible for the quality management of hospital infection in the area, standardizing the behavior of medical staff, and ensuring the implementation of various systems. The members of the prevention and control team regularly participated in the morning shift in the isolation ward, reported the management and operation of the hospital, and timely found, communicated and solved the problems.

\subsection{Layout Transformation and Process Improvement}

\subsubsection{Reasonable Layout and Clear Identification}

After the decision of experts, the isolation ward in our hospital will be located in three independent buildings, the original layout of the ward is mainly living areas and working areas, the open design of the ward and office areas. On the basis of the original ward, according to the infectious disease ward set standards for transformation, in strict accordance with the requirements of infectious disease area to be divided into three areas, four channels. Three zones: three zones are clean zone, potential pollution zone and pollution zone. Two channels: the patient channel, the staff channel. Each area is clearly marked. And after the transformation of the staff to work route, strict personnel access process management. Medical personnel from the clean area to the semi-contaminated area, and then into the ward, it is strictly required that entry into each level area must be in accordance with the provisions of changing clothes. The patient enters from the patient channel entrance and enters the ward through the patient channel. Each partition does not cross each other, goods area is dedicated. In addition, in order to minimize the number of irrelevant personnel entering and returning between the three areas, use walkie-talkie or special mobile phone, make full use of the mobile phone or WeChat function, timely understand the communication of the disease, but also to minimize the consumption of protective clothing and other protective supplies.

\subsubsection{Prevention and Control Supervision, Escort}

set up $24 \mathrm{~h}$ class control supervision, check the airtight sex of the protective equipment and safety, wear protective clothing off process in strict accordance 
with the requirements, responsible for all kinds of personnel (doctors, nurses, nursing workers, etc.) wearing and removing protective appliances throughout the process supervision, focusing on links and details of assistance and supervision to ensure effective protection. And install monitoring in the area where the protective appliances are removed to ensure the standardization of the removal, so as to "wear someone to remind, remove someone to supervise", and to wear and remove the protective appliances area garbage supervision, guide the orderly cleaning.

\subsection{Standard Disinfection Disposal}

\subsubsection{In the Early Stage of the Epidemic}

By consulting literature and discussing with hospital infection experts, practical disinfection methods were developed for stethoscope, sphygmomanometers, ECG monitors, infusion pump/micropump and other routine nursing supplies, fiber bronchoscopes and reusable protective goggles. Two video training sessions were held for the disinfection of fiberoptic bronchoscopes and other instruments and equipment, the key points of medical staff protection and psychological adjustment to ensure that everyone knew. Air disinfection is mainly based on ventilation. The ward is ventilated twice a day (9:00 AM to 09:40 AM, 3:00 PM to 3:40 PM) for a period of not less than 30 minutes, keep the patient warm and prevent cold.

\subsubsection{Mid-Term Epidemic Situation}

As protective supplies are gradually replenished, ultraviolet plasma disinfection instrument, bed unit ozone sterilizer, automatic disposable hand-drying and quick-drying disinfectant, and paper towel device are provided as standard. A watering can is also provided for spraying $2000 \mathrm{mg} / \mathrm{L}$ effective chlorine disinfectant to each other when the medical personnel finish working. The patient bed unit and bedside table were cleaned by one bed and one towel, and the bed towel was soaked and disinfected with $2000 \mathrm{mg} / \mathrm{L}$ chlorine disinfectant. The ward, treatment room, office, nurse station, and duty room are disinfected with a mobile ultraviolet plasma disinfection instrument, 60 minutes each time, 2 to 3 times a day. After invasive operations such as tracheal intubation, tracheotomy, and bronchoalveolar lavage, the surroundings of the patient, including the bed unit and surrounding instruments, are wiped and disinfected.

\subsubsection{Late Epidemic Situation}

The public health department of our hospital carried out sampling and testing on the environment of the isolated ward, including the items in the duty room, doctor's office, nurse's station, ward, floor and door handle, among which the sample on the bathroom floor of the isolated ward in our hospital showed positive nucleic acid. In view of this situation, our hospital has increased the disinfection management of the ward toilets, and carried out special quality improvement. Every day, the nursing workers are specially arranged to clean the bathroom floor with $2000 \mathrm{mg} / \mathrm{L}$ chlorine-containing disinfectant. 


\subsection{Orderly Protective Equipment Management}

The protective equipment management department is responsible for the selection, procurement, acceptance and storage management, establishes a dynamic management ledger, reports daily to the prevention and control security team, and according to the principle of "giving priority to protecting high-risk areas, high-risk operations and high-risk personnel", we should formulate standards for protective equipment, reasonable distribution of protective equipment, departments are prohibited from hoarding protective equipment. Isolation ward highlights the location of protective equipment, in accordance with the order of wear of protective equipment, the protective equipment required to enter the ward will include protective clothing, isolation clothing, protective goggles, face screen, N95 mask, disposable hat, surgical gloves, Shoe covers, boot covers, etc, are packaged and placed according to the model and placed in the corresponding finishing box, the name of the article is clearly marked on the outside of the box. Make a diagram of the putting on and taking off process, and post it in a conspicuous position, which is convenient for medical staff to self-manage and self-check whether the protective clothing meets the requirements. For a limited number of special protective equipment, such as a positive pressure headgear, it is managed by the head nurse who is responsible for the overall coordination of the COVID-19 Critical Care Center, which can be distributed only after the head of the treatment center evaluates the risk and agrees.

\subsection{Solve the Worries of Frontline Personnel}

On February 7th, the State Council issued the "Notice on Ensuring the Protection of First-line Medical Staff and Their Families" in response to thecovid-19 epidemic prevention and control mechanism, combined with the actual situation of the hospital [7], our hospital can solve the worries of front-line medical staff from the following aspects: 1) Security guarantee: rational deployment of human resources and shift arrangement. In the early stage of the epidemic, due to the shortage of protective supplies, the daily shift time was $5-7$ hours to balance the use of protective supplies. With the supplement of protective supplies in place, the working time of nursing staff should be shortened in a timely manner to ensure adequate rest time. Nursing staff in high-risk areas (isolation ward, isolation intensive care unit, fever clinic, etc.) should be rotated for 4 to 6 hours per shift. 2) Life security: Pay attention to the health of medical personnel and carry out active health monitoring, including body temperature and respiratory symptoms. Provide nutritious meals three times a day, and provide relatively independent resting places for first-line medical staff (not close contacts) in high-risk positions in or near the hospital to ensure the supply of basic daily necessities and ensure adequate sleep for medical staff. 3) Psychological protection: take the initiative to care about the front-line medical personnel from a variety of perspectives, especially not to discriminate against the front-line medical personnel, and take the front-line medical personnel as the key psychological intervention 
object, through different forms of heart-to-heart counseling, reduce their psychological anxiety, give positive spiritual care.

\section{Effect}

From January 16, 2020 to March 23, 2020, our hospital has set up 5 isolated wards and one COVID-19 intensive care center. More than 700 medical personnel were deployed to participate in the frontline of epidemic prevention and control. There were more than 100 outside medical personnel who joined us. More than 240 patients with covid-19 were treated, including more than 100 critically ill patients, to carry out the nasopharyngeal swabs collected more than 3000 times, ECMO (Extracorporeal Membrane Oxygenation, ECMO) 3 cases, CRRT (Continuous Renal Replacement Therapy, CRRT) more than 10 people, invasive mechanical ventilation more than 10 people, noninvasive ventilation more than 80 people, high flow oxygen therapy more than 80 people, the hospital general ward in hospital patients per day is about 400 - 700 more than person. During the epidemic period, there was not one case of nosocomial infection among medical staff, patients and family members in the fever outpatient clinic, isolation ward and general ward. It not only guarantees the smooth progress of the epidemic prevention and control work, but also guarantees the safety of the patients in the general ward.

\section{Summary}

In May 2018, the General Office of the National Health Commission issued a notice on further strengthening the prevention and control of infection in medical institutions, which mentioned that the prevention and control of hospital infection is the "main line" throughout the diagnosis and treatment activities, the "bottom line" to ensure the safety of patients, and the "red line" to practice according to law [8]. However, since the occurrence of COVID-19, hospital infections of COVID-19 have been reported among medical staff [9], which has greatly affected the physical and mental health of medical staff. In the face of this unknown new virus with high transmissibility, although China has issued a series of diagnosis, treatment and prevention and control guidelines according to the characteristics of COVID-19, the accumulated measures of hospital infection prevention and control are faced with severe challenges in practice, and there are still many difficulties to be solved in the practice of hospital infection prevention and control. Our hospital implemented a comprehensive quality management model to formulate detailed measures in terms of personnel management, comprehensive training, prevention and control plans, environmental protection, etc., scientific planning, meticulous implementation, seamless docking, and scientific management of COVID-19 safety protection, which has been achieved in stages more than 700 front-line medical staff of the epidemic had zero infection targets, which ensured the physical and mental health of the front-line medical staff and ensured the smooth implementation of the COVID-19 epidemic. The innovation 
of this paper is to apply this model to the quality management of hospitals during the epidemic period for the first time based on the concept of total quality management. Based on the actual situation of this hospital, the requirements of all aspects during the epidemic period are fully taken into account, dynamic adjustment is made, and 24-hour quality supervision is conducted throughout the whole process to escort the battle against the epidemic. In the face of the sudden epidemic, we must keep exploring and forging ahead to overcome it.

\section{Conflicts of Interest}

The authors declare no conflicts of interest regarding the publication of this paper.

\section{References}

[1] Qiang, F. (2020) Practice of Infection Prevention and Control in Medical Institutions Based on COVID-19 Epidemic Response. Chinese Journal of Nosocomial Infection, 30, 1121-1124.

[2] Li, X.M. (2006) The Application of Whole-Process Quality Management in Nursing Quality Control Management. Journal of Nursing of Chinese PLA, No. 7, 86-87.

[3] Lei, Y. (2018) Evaluation of the Construction and Implementation of Hospital Quality Management System Based on Total Quality Management (TQM) Concept. University of South China, Hengyang.

[4] Chen, C.X. and Li, M.X. (2006) The Whole Process Quality Management of the Delivery of Sterile Materials in the Central Supply Room. Journal of Nursing Management, 5, 35-36.

[5] Lei, Y. (2004) Practice and Experience of Medical Quality Management in SARS Designated Hospitals. Chinese Health Resources, 2, 91-92.

[6] General Office of the National Health and Health Commission (2020) Notice of the General Office of the National Health and Health Commission on Printing and Distributing the "COVID-19 Diagnosis and Treatment Plan (Trial Implementation Seventh Edition)".

http://www.nhc.gov.cn/xcs/zhengcwj/202003/46c9294a7dfe4cef80dc7f5912eb1989.s $\underline{\mathrm{html}}$

[7] General Office of National Health Commission (2020) Notice on Ensuring the Protection of First-Line Medical Staff and Their Families.

http://www.nhc.gov.cn/xcs/zhengcwj/202002/d919a2a8313144b399065e6eb825e9b1. $\underline{\text { shtml }}$

[8] General Office of the National Health and Health Commission (2019) Notice of the General Office of the National Health and Health Commission on Further Strengthening Infection Prevention and Control in Medical Institutions. http://www.nhc.gov.cn/yzygj/s7659/201905/d831719a5ebf450f991ce47baf944829.sht $\underline{\mathrm{ml}}$

[9] Wang, D., Hu, B., Hu, C., et al. (2020) Clinical Characteristics of 138 Hospitalized Patients with 2019 Novel Coronavirus-Infected Pneumonia in Wuhan, China. Journal of the American Medical Association, 323, 1061-1069.

https://doi.org/10.1001/jama.2020.1585 